\title{
Estudio transversal: pérdida auditiva causada por ruido en el personal de la aviación militar ecuatoriana
}

| Fecha de recibido: 24 de enero del 2021 | Fecha de aprobación: 9 de abril del 2021 |

\section{Álvaro Guillermo Ortiz Rodríguez}

Magíster en Gestión Ambiental

Fuerza Aérea Ecuatoriana Ecuador

Rol de investigador: experimental y escritura https://orcid.org/0000-0002-9277-6097

$\bowtie$ alvaroortizr0@gmail.com 


\section{Estudio transversal: pérdida auditiva causada por ruido en el personal de la aviación militar ecuatoriana}

\section{Cross-sectional Study: Noise-induced Hearing Loss in Ecuadorian Military Aviation Personnel}

Resumen: La hipoacusia como condición de salud crónica ocupa uno de los primeros lugares en países como Norteamérica, superando a la diabetes y el cáncer, motivo por el cual, desde hace décadas sus Fuerzas Armadas iniciaron estudios pormenorizados con el fin de implementar medidas preventivas adecuadas; no obstante, la aviación militar tiene sus particularidades debido a que las principales fuentes de ruido son sus propias aeronaves. Dicho lo anterior, el objetivo de esta investigación es correlacionar la pérdida auditiva con los factores: edad, actividad o rango, analizando las audiometrías de 73 miembros de la aviación ecuatoriana y verificar su estado actual de acuerdo con los umbrales de audición de frecuencias. Los resultados indican que la mayor cantidad de afectados se presentan en el personal de tropa de sexo masculino de 30 a 44 años de edad. Sin embargo, la variabilidad de los niveles de hipoacusia no evidenció la existencia de una relación directa entre pérdida auditiva y el incremento en la edad, tiempo de servicio, actividad inherente al tipo de aeronave, o rango del individuo, por lo que no se puede aseverar que para el caso de la Institución la hipoacusia sea producida exclusivamente por las condiciones laborales y, más bien, puede ser producto de antecedentes clínicos de la persona. En consecuencia, se evidencia un eficaz programa preventivo y medidas de mitigación adecuadas, referentes a la seguridad y salud ocupacional. Aunque es imprescindible realizar estudios más detallados, siendo más enfáticos en las Bases Aéreas donde se despliegan aeronaves de combate y de ala rotatoria.

Palabras clave: contaminación acústica; hipoacusia; mantenimiento de aeronaves; ruido aeronáutico; salud ocupacional; seguridad industrial.

Abstract: Hearing loss holds an important place among chronic health conditions in North American countries, surpassing diabetes and cancer. Therefore, for decades, the Armed Forces in these nations began detailed studies in order to implement adequate preventive measures. However, military aviation has the peculiarity that the main sources of noise are aircraft themselves. With that being said, this research seeks to correlate hearing loss with the variables age, activity, and rank within military aviation, by analyzing audiometry reports of 73 members of the Ecuadorian aviation and later verifying their current status regarding frequency hearing thresholds. Our results show that the biggest number of affected individuals correspond to male personnel aged 30-44. However, the variability of hearing loss levels did not signal a direct relationship between this condition and an increase in age, service time, activity and type of aircraft, or the rank held by participants. Hence, it is not possible to assert that, in the case of the studied institution, hearing loss is produced exclusively by working conditions, but rather by individuals' clinical history. Consequently, there is evidence of an effective preventive program and adequate mitigation measures in occupational health and safety. Despite this, it is essential to carry out additional in-depth studies in airbases where combat and rotary-wing aircraft are deployed.

Keywords: Noise pollution; hearing loss; aircraft maintenance; aeronautical noise; occupational safety and health; industrial Security.

Resumo: A hipoacusia como condição de saúde crônica ocupa um dos primeiros lugares em países da América do Norte, superando a diabetes e o câncer, motivo pelo qual, desde há décadas que as suas Forças Armadas iniciaram estudos aprofundados a fim de implementar medidas preventivas adequadas; No entanto, a aviação militar tem as suas particularidades, uma vez que as principais fontes de ruído são as suas próprias aeronaves. Dito isto, o objetivo desta investigação é correlacionar a perda auditiva com os fatores: idade, atividade ou intervalo, analisando as audiometrias de 73 membros da aviação equatoriana e verificando seu estado atual de acordo com os limiares de audição de frequências. Os resultados indicam que o maior número de pessoas afetadas são apresentados no pessoal de tropa do sexo masculino de 30 a 44 anos de idade. No entanto, a variabilidade dos níveis de hipoacusia não evidenciou a existência de uma relação direta entre perda auditiva e aumento da idade, tempo de serviço, atividade inerente ao tipo de aeronave, ou categoria do indivíduo, pelo que não se pode afirmar que, no caso da instituição, a hipoacusia é produzida exclusivamente pelas condições de trabalho e, em vez disso, pode ser produto de antecedentes clínicos da pessoa. Em consequência, evidencia-se um eficaz programa preventivo e medidas de mitigação adequadas, referentes à segurança e saúde ocupacional. Apesar de ser imprescindível realizar estudos mais detalhados, sendo mais enfáticos nas bases aéreas onde são desdobradas aeronaves de combate e de asa rotatória.

Palavras-chave: poluição acústica; hipoacusia; manutenção de aeronaves; ruído aeronáutico; saúde ocupacional; segurança industrial. 
La hipoacusia o pérdida auditiva es una de las condiciones de salud crónica más común en Norteamérica, superando a la diabetes y el cáncer (Yankaskas et al., 2017). Las Fuerzas Armadas estadounidenses a finales de la Segunda Guerra Mundial iniciaron medidas preventivas en el ámbito global, debido al alto índice de afectaciones en la percepción sonora de sus combatientes luego de la intervención en dicho conflicto, lo cual inclusive, llegó a ser motivo de millonarias demandas luego de operaciones más recientes como las de Irak y Afganistán. Por ello, las regulaciones implantadas en el país del norte han servido de base para la expedición de normativas en otros países (Kerr et al., 2017), que en la actualidad están encabezadas fundamentalmente por la Organización Mundial de la Salud (oms) (Héroux et al., 2018). Las principales medidas adoptadas se centran en la utilización de elementos de protección auditiva, sean simples o dobles, como los tipo orejera, tapones de inserción o el uso combinado de ambos (Gwenolé NEXER, 2013), pese a que internacionalmente no existe una estandarización para el cálculo de la atenuación proporcionada por estos dispositivos, existen diferencias sustanciales en los métodos aplicados (Rodríguez, 2003), que dependen de si estos están sometidos a bajas o altas frecuencias (Coello et al., 2017). Aunque eventualmente se puede optar por otro tipo de barreras acústicas que no son aplicables en la generalidad de los casos (HernándezSánchez, 2013).

Las alteraciones congénitas, tumoraciones, envejecimiento, exposición a sustancias ototóxicas y al ruido, entre otros, constituyen los componentes causantes de la hipoacusia (Olarieta et al., 2015) y de la denominada pérdida auditiva oculta (Tepe et al., 2017). De modo que, la exposición a la contaminación acústica es uno de los factores más comunes en las operaciones militares y objeto de diversas investigaciones en el campo militar, debido a que los niveles de ruido alcanzan valores importantes, como es el caso de las armas de fuego que superan los $130 \mathrm{~dB}$ (Heupa et al., 2011). Un estudio realizado en la Marina Real Noruega en el 2013, basado en pruebas de audiometría obtuvo un total de 190 individuos con pérdida auditiva, equivalente al $31,4 \%$ de la muestra evaluada, de los cuales en su mayoría correspondía a personal de navegantes y personal encargado del cuarto de máquinas, íntimamente relacionado a la edad del individuo (Irgens-Hansen et al., 2015).

En el 2012, el personal militar estadounidense realizó una investigación de pérdida de audición autoinformada, validada con estudios audiométricos, en la cual el 7,5\% de los 48.540 casos estudiados reportaron una nueva pérdida de audición, la mayoría de individuos de sexo masculino nacidos antes de 1970 y pertenecientes al Ejército, Marina y Guardia Costera (Wells et al., 2015). En otro estudio, se evaluó a veteranos de guerra americanos luego de aproximadamente tres años de haberse separado de las filas milicianas, entre ellos, el $15 \%$ de participantes fueron identificados con pérdida de audición de baja a media frecuencia, mientras que el $25 \%$ lo reflejó en altas frecuencias, encontrando los casos más críticos en edades superiores a 41 años y una diferencia casi imperceptible en exmiembros de la Fuerza Aérea, frente a las otras ramas; por el contrario, sí hubo diferencias significativas en aquellos individuos con más de 20 años de servicio (Gordon et al., 2017).

Los procedimientos y evaluaciones de las afectaciones del ruido en los combatientes han evolucionado y perfeccionado con el tiempo, buscando ser más precisos y cercanos a la realidad, como lo constituye la propuesta de caracterización de enfermedades agudas adquiridas a corto plazo por parte de la población militar (Hecht et al., 2019), la cual promueve la realización de pruebas audiométricas antes e inmediatamente después de la exposición a ruidos continuos, como los generados por buques, tanques, ruidos impulsivos como los de un rifle o explosiones que pueden alcanzar los $180 \mathrm{~dB}$. La aplicación de estos test no es del todo factible, debido a los altos costos, ya que la audiometría debe realizarse en exteriores y en condiciones totalmente diferentes a las convencionales.

En actividades similares llevadas a cabo por la fuerza pública, policía y bomberos, existen investigaciones como la realizada en Córdoba, España, en la cual, según los criterios de Klockhoff, un total de 207 
trabajadores equivalentes al 53,2 \% resultaron con audiogramas anormales, adicional se determinó que el 35,7 \% de ellos resultaron ser casos de hipoacusia neurosensorial (Álvarez-Fernández et al., 2016).

En la rama de la aviación, específicamente en la tripulación de aeronaves, la Fuerza Aérea Colombiana en el 2015, determinó que el $5 \%$ de un total de 1.629 pacientes presentó hipoacusia inducida por ruido, con mayor afectación en el oído izquierdo, diagnóstico que aparece a partir de los 5 años de servicio del personal; no obstante, se encontraron niveles importantes de pérdida auditiva en población joven determinando que el ambiente laboral no es la causa principal y sí, se asocia a factores externos como el uso de audífonos con presiones sonoras superiores a $85 \mathrm{~dB}$ ( $\mathrm{Ca}$ puto \& Correa, 2018). Por su parte, en la aviación del Ejército Ecuatoriano en el 2016, se estudió a un total de 112 individuos, entre pilotos, ingenieros de vuelo y mecánicos, de los cuales el 19,6\% presentó hipoacusia unilateral y el $16 \%$ hipoacusia bilateral, mientras que la hipoacusia leve, moderada y severa fue del $15,2 \%, 7,1 \%$ y $1,8 \%$, respectivamente. En cuanto al grado militar, los oficiales de mayor tiempo de servicio, y por ende, con más horas de vuelo, resultaron ser de los más afectados (Carpio-Ayora \& Álvarez-Pesantez, 2017).

La Fuerza Aérea Ecuatoriana (FAE), a diferencia del Ejército, opera con aeronaves de diferente tipo, esto es, de entrenamiento, ala rotatoria, transporte, combate a turbohélice y supersónicas, estas últimas, superan los $110 \mathrm{~dB}$ al momento del despegue (Corbalán et al., 2019). Es decir, no solamente está expuesta la tripulación, sino también el personal de apoyo en tierra, por lo que esta investigación pretende determinar la relación existente entre el grado de afectación de la capacidad auditiva y variables como edad o tiempo de servicio, rango y actividad dentro de la institución, a fin de fortalecer las medidas de prevención existentes y focalizarlas, de ser necesario. De igual forma, podrán implementarse medidas de mitigación oportunas, reduciendo ostensiblemente la afectación del personal, y así, constituir una política generalizada en todos los países, en especial europeos, en donde se responsabiliza a los empleadores de todo lo relacionado con los procedimientos de evaluación de riesgos en el ambiente laboral (Stolk et al., 2012), lo que involucra un gran reto para la aviación militar, al estar sujeta a innumerables externalidades que directa e indirectamente influyen en el desarrollo normal de las actividades.

\section{Metodología}

Se recopilaron las audiometrías realizadas entre el 2018 y el 2019 -controles anuales y bianuales-, del personal militar al que de acuerdo con los registros clínicos de la FAE, se le diagnosticó algún tipo de afección auditiva, hipoacusia de alta frecuencia, percepción de posible pérdida de audición, y personal que respecto a sus funciones, se le recomendó el uso de protectores auditivos. La muestra suma un total de 73 miembros, entre oficiales y aerotécnicos.

A partir de los audiogramas se obtuvieron y tabularon los valores de los umbrales auditivos de las frecuencias $500 \mathrm{~Hz}, 1.000 \mathrm{~Hz}, 2.000 \mathrm{~Hz}$ y $3.000 \mathrm{~Hz}$, con el objeto de calcular el porcentaje de pérdida de audición monoaural mediante la sumatoria de dichos umbrales (sum), de acuerdo con las tablas de conversión establecidas en la normativa española (Ministerio de Trabajo y Asuntos Sociales, 2000), cuyo extracto se visualiza en la tabla 1. Luego, se calculó la pérdida de audición binaural (Pab), aplicando la ecuación (1) y clasificando el tipo de discapacidad conforme a la normativa antes citada.

La pérdida de audición monoaural y binaural fue contrastada con las variables: edad, rango y actividad según el tipo de aeronave, para determinar la prevalencia de afectados. Así mismo, a través de técnicas de estadística inferencial en lenguaje $\mathrm{R}$ para un nivel de confianza del $95 \%(\alpha=0,05)$, se evaluó la posible relación existente entre dichas variables y los porcentajes de hipoacusia obtenidos, aplicando un análisis Anova y un test Kruskal-Wallis. 
Tabla 1

Pérdida auditiva monoaural

\begin{tabular}{c|c|c|c|c|c|c|c|c|c}
\hline sUm & $\%$ & sUm & $\%$ & sUm & $\%$ & sUm & $\%$ & sUm & $\%$ \\
\hline 100 & 0,0 & 130 & 11,2 & 160 & 22,5 & 190 & 33,8 & 220 & 45,0 \\
\hline 105 & 1,9 & 135 & 13,1 & 165 & 24,4 & 195 & 35,6 & 225 & 46,9 \\
\hline 110 & 3,8 & 140 & 15,0 & 170 & 26,2 & 200 & 37,5 & 230 & 48,9 \\
\hline 115 & 5,6 & 145 & 16,9 & 175 & 28,1 & 205 & 39,4 & 235 & 50,5 \\
\hline 120 & 7,5 & 150 & 18,8 & 180 & 30,0 & 210 & 41,2 & 240 & 52,5 \\
\hline 125 & 9,4 & 155 & 20,6 & 185 & 31,9 & 215 & 43,1 & 245 & 54,4 \\
\hline
\end{tabular}

Fuente: Ministerio de Trabajo y Asuntos Sociales (2000).

$P a b=\left(\frac{5 *(\% \text { pérdida mejor oído })+\% \text { pérdida peor oído }}{6}\right)$

Donde:

Pab es la pérdida de audición binaural en \%

\section{Resultados}

Los 73 miembros de la FAE corresponden en su totalidad al sexo masculino, de los cuales cinco son oficiales y 68 aerotécnicos, con rango de edad entre los 23 y 50 años, y un tiempo de servicio de dos a 26 años, el $32,88 \%$ del personal evaluado realiza actividades relacionadas con aeronaves supersónicas, conforme se observa en la tabla 2.

Tabla 2

Frecuencia del personal evaluado de acuerdo con las variables

\begin{tabular}{l|l|c|c}
\hline \multirow{4}{*}{ Edad } & \multicolumn{1}{|c|}{ Variable } & Frecuencia & Porcentaje \\
\cline { 2 - 4 } & Joven 20-29 & 6 & 8,22 \\
\cline { 2 - 4 } & Adulto joven 30-44 & 43 & 58,90 \\
\cline { 2 - 4 } & Adulto mayor 45-65 & 24 & 32,88 \\
\hline \multirow{3}{*}{ Rango } & Oficiales & 5 & 6,85 \\
\cline { 2 - 4 } & Aerotécnicos & 68 & 93,15 \\
\hline \multirow{4}{*}{ Aeronave } & Ala rotatoria & 14 & 19,18 \\
\cline { 2 - 4 } & Combate turbohélice & 7 & 9,59 \\
\cline { 2 - 4 } & Entrenamiento & 2 & 2,74 \\
\cline { 2 - 4 } & Supersónico & 24 & 32,88 \\
\cline { 2 - 4 } & Transporte & 17 & 23,29 \\
\cline { 2 - 4 } & Otros & 9 & 12,33 \\
\hline
\end{tabular}

Fuente: elaboración propia.

\section{Pérdida de audición monoaural y binaural}

De acuerdo con las pruebas audiométricas, el 71,23\% de la muestra estudiada refleja una condición auditiva normal, mientras que el 20,55\% presenta hipoacusia monoaural y el $28,77 \%$ hipoacusia binaural, conforme se visualiza en la figura 1.

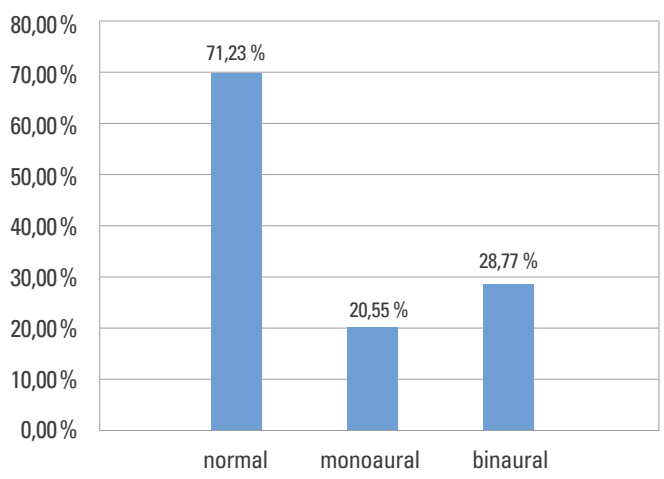

Figura 1. Presencia de hipoacusia en el personal evaluado Fuente: elaboración propia.

\section{Pérdida de audición monoaural y binaural según edad}

En cuanto a la pérdida auditiva del oído derecho, conforme se muestra en la tabla 3, el 19,18\% de personas en su mayoría adulto joven (10,96\%), presenta pérdida auditiva, con el mayor valor correspondiente al 41,2\% de deficiencia en este rango de edad, lo cual se verifica visualmente en la figura 2. Por otro lado, para el caso del oído izquierdo, el mayor porcentaje de pérdida auditiva con el 39,4\%, se presenta en el rango de 30 a 44 años de edad, sumando un total de 15 casos con esta condición correspondientes al 20,55\%, de los cuales el $13,70 \%$ está en el rango de edad intermedio.

La afectación binaural leve con el $27,40 \%$ y moderada con el 1,37\%, del total de casos analizados, en su gran mayoría se presenta entre los 30 y 44 años de edad, reflejando una deficiencia auditiva máxima del 39,7\% considerada como clase de discapacidad tipo 3.

El análisis estadístico Anova realizado en $\mathrm{R}$, para un nivel de confianza del $95 \%$, refleja que el incremento en la edad no incide directamente en los niveles de hipoacusia monoaural, obteniendo un $p$-value 
de 0,663 , lo cual es ratificado con el test de KruskalWallis, con un $p$-value de 0,9434 . De igual forma, no existe incidencia del aumento en la edad sobre los porcentajes de pérdida de audición binaural, obteniendo así un $p$-value de 0,683 para el análisis Anova y un $p$-value de 0,8588 para el test de Kruskal-Wallis.

Tabla 3

Pérdida de audición monoaural y binaural según edad

\begin{tabular}{|c|c|c|c|c|c|c|}
\hline & \multicolumn{2}{|c|}{$\%$ Afectados } & Edad & $\begin{array}{c}\% \text { en } \\
\text { relación }\end{array}$ & $\begin{array}{l}\text { Tiempo de } \\
\text { servicio }\end{array}$ & $\begin{array}{c}\% \text { pérdida } \\
\text { auditiva }\end{array}$ \\
\hline \multirow{6}{*}{ 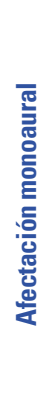 } & \multirow{3}{*}{$O D$} & \multirow{3}{*}{19,18} & Joven $20-29$ & 1,37 & 2 & 1,9 \\
\hline & & & $\begin{array}{l}\text { Adulto } \\
\text { joven 30-44 }\end{array}$ & 10,96 & $7-21$ & $5,6-41,2$ \\
\hline & & & $\begin{array}{l}\text { Adulto } \\
\text { mayor } 45-65\end{array}$ & 6,85 & $23-26$ & $1,9-24,4$ \\
\hline & \multirow{3}{*}{ 이 } & \multirow{3}{*}{20,55} & Joven 20-29 & 2,74 & $2-4$ & $5,6-7,5$ \\
\hline & & & $\begin{array}{l}\text { Adulto } \\
\text { joven 30-44 }\end{array}$ & 13,70 & $12-21$ & $1,9-39,4$ \\
\hline & & & $\begin{array}{l}\text { Adulto } \\
\text { mayor 45-65 }\end{array}$ & 4,11 & $22-24$ & $1,9-11,2$ \\
\hline \multirow{6}{*}{ 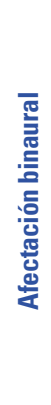 } & \multirow{3}{*}{ Leve } & \multirow{3}{*}{27,40} & Joven 20-29 & 2,74 & $2-4$ & $2,5-7,5$ \\
\hline & & & $\begin{array}{l}\text { Adulto } \\
\text { joven 30-44 }\end{array}$ & 16,44 & $7-21$ & $3,8-15,0$ \\
\hline & & & $\begin{array}{l}\text { Adulto } \\
\text { mayor } 45-65\end{array}$ & 8,22 & $23-26$ & $3,8-11,2$ \\
\hline & \multirow{3}{*}{ Moderada } & \multirow{3}{*}{1,37} & Joven 20-29 & --- & --- & --- \\
\hline & & & $\begin{array}{l}\text { Adulto } \\
\text { joven 30-44 }\end{array}$ & 1,37 & 15 & 39,7 \\
\hline & & & $\begin{array}{l}\text { Adulto } \\
\text { mayor 45-65 }\end{array}$ & --- & -- & -- \\
\hline
\end{tabular}

Fuente: elaboración propia.

\section{Pérdida de audición según actividad}

En cuanto a la pérdida de audición monoaural según el tipo de avión al cual el personal realiza el mantenimiento, tanto para el oído derecho como para el oído izquierdo, el mayor porcentaje de afectados se da en la aviación supersónica con el 6,85\% y 8,22 \%, como se observa en la tabla 4, cuyos porcentajes de hipoacusia más altos $(41,2 \%$ y $39,4 \%)$ se presentan en las aeronaves de ala rotatoria como se visualizan en la figura 3.

La mayor cantidad de afectados con hipoacusia binaural se registra en el personal de actividades inherentes a las aeronaves supersónicas con el 12,33\% de pacientes, seguido de las actividades correspondientes al ala rotatoria con el 6,85\% (figura 4), evidenciándose una pérdida auditiva del $39,7 \%$ en un elemento especializado en helicópteros (figura 5).

Tabla 4

Pérdida de audición monoaural y binaural según actividad

\begin{tabular}{l|c|c|c|c}
\hline \multirow{2}{*}{ Aeronave } & \multicolumn{4}{|c}{ \% respecto al total } \\
\hline Ala rotatoria & $\begin{array}{c}\text { Afectación } \\
\text { OD }\end{array}$ & $\begin{array}{c}\text { Afectación } \\
\text { 0I }\end{array}$ & \multicolumn{2}{|c}{ Afectación binaural } \\
\cline { 4 - 5 } & 6,85 & 5,48 & 5,48 & 1,37 \\
\hline $\begin{array}{l}\text { Combate } \\
\text { turbohélice }\end{array}$ & 1,37 & 1,37 & 2,74 & --- \\
\hline Entrenamiento & 0,00 & 1,37 & 1,37 & --- \\
\hline Supersónico & 6,85 & 8,22 & 12,33 & --- \\
\hline Transporte & 2,74 & 1,37 & 2,74 & --- \\
\hline Otros & 1,37 & 2,74 & 2,74 & --- \\
\hline Total & $\mathbf{1 9 , 1 8}$ & $\mathbf{2 0 , 5 5}$ & $\mathbf{2 7 , 4 0}$ & $\mathbf{1 , 3 7}$ \\
\hline
\end{tabular}

Fuente: elaboración propia.

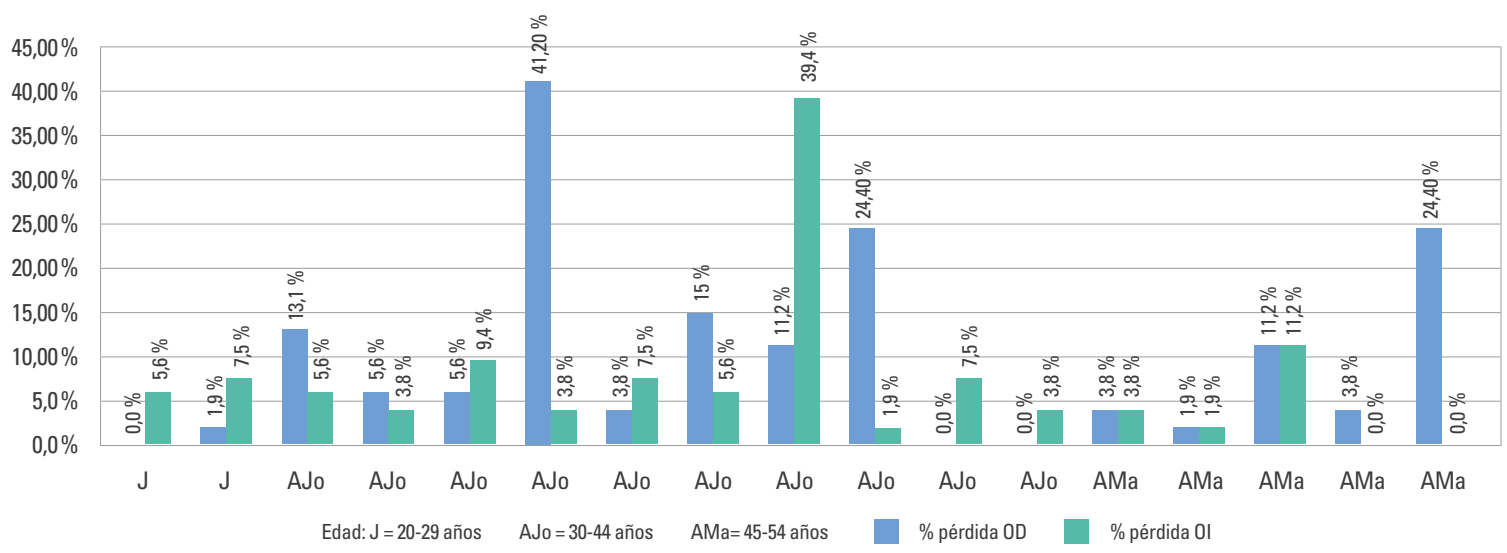

Figura 2. Pérdida de audición monoaural, oído derecho e izquierdo Fuente: elaboración propia. 


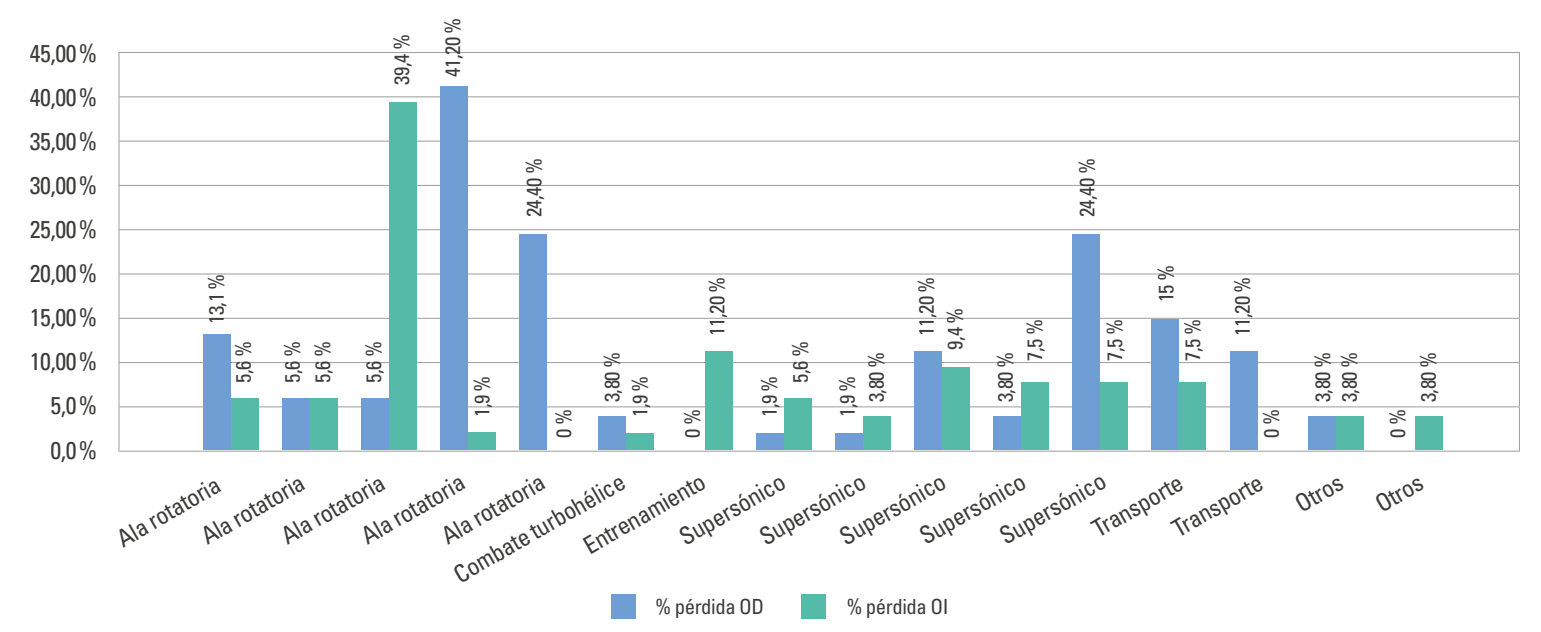

Figura 3. Pérdida de audición monoaural según actividad Fuente: elaboración propia.

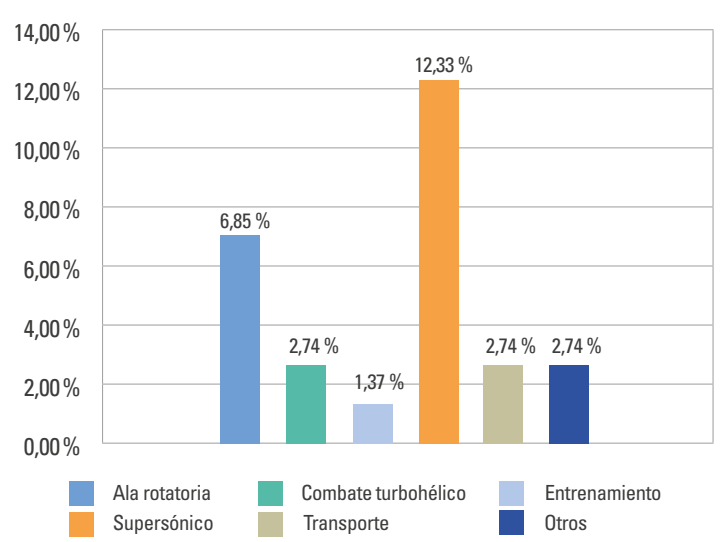

Figura 4. Afectados audición binaural según actividad y aeronave Fuente: elaboración propia.

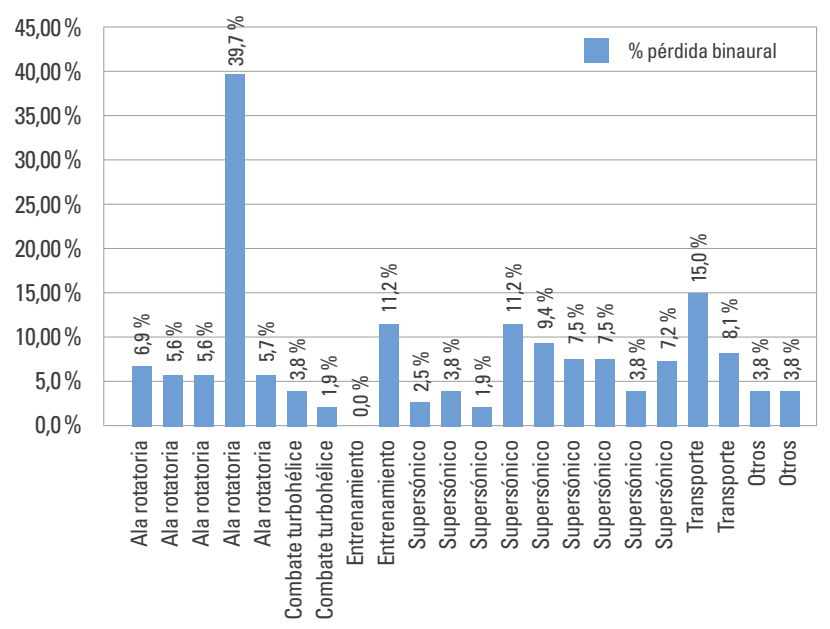

Figura 5. Afectación binaural según actividad y aeronave Fuente: elaboración propia.
La estadística inferencial demuestra que el tipo de actividad no es determinante en los niveles de pérdida auditiva monoaural, obteniendo un $p$-value de 0,221 y 0,4515 para el análisis Anova y el test Kruskal-Wallis, respectivamente. Similares resultados se obtienen para la hipoacusia binaural, ya que no existe evidencia significativa de incidencia de la actividad desempeñada con un $p$-value de 0,482 y 0,5013 para los parámetros estadísticos.

\section{Pérdida de audición binaural según rango}

Conforme se advierte en la tabla 5, los afectados con hipoacusia en su mayoría son aerotécnicos, que corresponden al $27,40 \%$ del personal evaluado, existe un solo caso para oficiales, los porcentajes de hipoacusia se visualizan en la figura 6 y resulta el mayor valor para un señor aerotécnico con el 39,70\%.

Tabla 5

Afectados con pérdida de audición binaural según rango

\begin{tabular}{l|c|c|c|c}
\hline & & \multicolumn{3}{|c}{$\%$ respecto del total } \\
\hline Rango & Afectados & Leve & Moderada & Total \\
\hline Oficiales & 1 & 1,37 & 0,0 & 1,37 \\
\hline Aerotécnicos & 20 & 26,03 & 1,37 & 27,40 \\
\hline Total & $\mathbf{2 1}$ & $\mathbf{2 7 , 4 0}$ & $\mathbf{1 , 3 7}$ & $\mathbf{2 8 , 7 7}$ \\
\hline
\end{tabular}

Fuente: elaboración propia. 


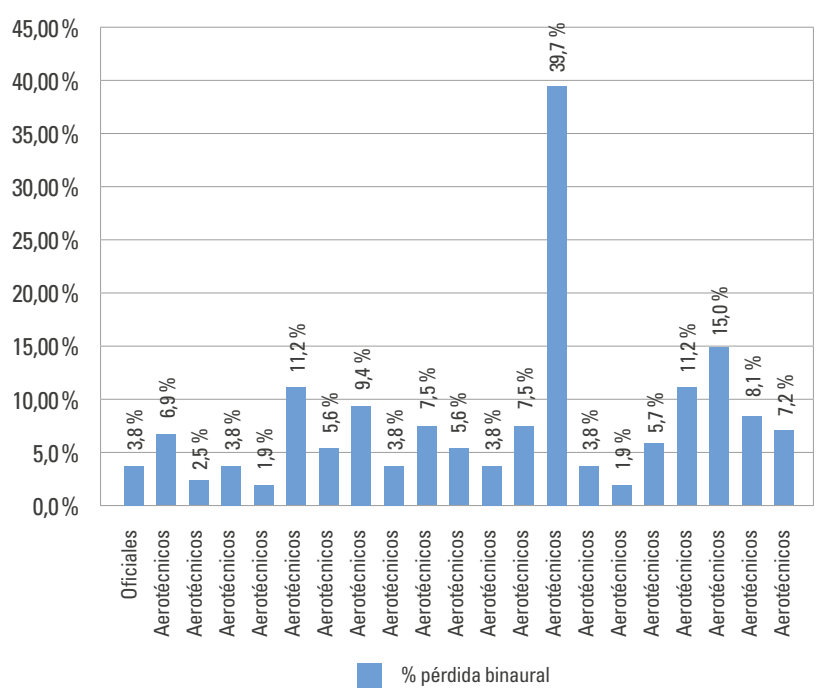

Figura 6 . Afectación binaural según rango

Fuente: elaboración propia.

\section{Discusión}

De acuerdo con los resultados obtenidos, los casos de hipoacusia para el oído izquierdo y derecho son similares con el 20,55\% y el 19,18\%, respectivamente. Para este último se presenta el mayor valor de pérdida auditiva con el $41,2 \%$, estos resultados no son compatibles con el estudio de Caputo y Correa (2018), ya que este se realizó exclusivamente a personal de tripulación, es decir, para el personal de pilotos; la fuente emisora principal de ruido la constituyen los motores, ubicados al lado izquierdo de la cabina, y por consiguiente, la hipoacusia monoaural se presenta en ese oído.

El mayor valor de afectación binaural se presenta en la edad intermedia de 30 a 44 años; sin embargo, estadísticamente y debido a la variabilidad de los resultados, el incremento en la edad no es determinante en los niveles de hipoacusia, contrario a los resultados obtenidos en la aviación del Ejército Ecuatoriano. Así mismo, el porcentaje de afectados con hipoacusia binaural del presente estudio, que asciende al 28,77\%, supera el porcentaje del $16 \%$ obtenido en la investigación de la Fuerza Terrestre. Los motivos principales de tal diferencia pueden ser antecedentes clínicos del personal analizado, pero sobre todo, los trabajos rotativos del personal en los diferentes tipos de aeronaves, que es común en la Fuerza Aérea.

La prevalencia de hipoacusia en esta investigación supera el valor del $5 \%$, obtenido por Caputo y Correa (2018) para el personal de tripulantes de la aviación colombiana, sector que en el caso ecuatoriano no presenta afectación auditiva, pues los casos de pérdida en la audición corresponden al personal de oficiales y aerotécnicos de mantenimiento de aeronaves.

La mayor cantidad de afectados con hipoacusia binaural corresponde a las especialidades relacionadas con la aviación supersónica, seguida de la aviación con ala rotatoria, aunque conforme al Anova, los niveles de afectación no guardan relación directa con el tipo de aeronave, es así que el mayor grado de afectación del 39,7 \% para un señor aerotécnico de 39 años de la especialidad de helicópteros, no puede ser considerado determinante, es un valor atípico que constituye un caso de discapacidad moderada y corresponde al 1,37\% del total de la muestra, frente al 7,1\% obtenido por Carpio-Ayora y Álvarez-Pesantez (2017) en el caso de Aviación del Ejército Ecuatoriano, en el que incluso, se presentaron casos de discapacidad severa, por lo que se puede deducir que los programas preventivos, controles médicos continuos y medidas de mitigación respecto al factor ruido, han sido eficaces en la Fuerza Aérea.

\section{Conclusiones}

La hipoacusia causada por ruido en el campo de la aviación depende de muchos factores como la edad y el tiempo de servicio, sin embargo, para la aviación militar ecuatoriana los casos que se han presentado resultan ser excepcionales al evidenciar que no dependen del incremento en los años, que está íntimamente relacionada con el periodo de permanencia en la institución, por lo que la pérdida auditiva manifestada en el personal de la Fuerza Aérea no está ligada directamente a la actividad laboral, pudiendo deberse más bien a asuntos externos, así como a antecedentes clínicos especiales. 
De igual forma, no existe evidencia suficiente para aseverar que la pérdida en la audición dependa del rango o actividad específica del oficial o aerotécnico, sin existir diferencias significativas entre el oído derecho y el izquierdo, presentándose hechos aislados en el caso de helicópteros y aviones supersónicos, hacia los cuales podrían focalizarse y fortalecerse las medidas preventivas y correctivas.

El programa preventivo y medidas de mitigación respecto al factor ruido han sido eficaces en la aviación militar ecuatoriana, ya que al considerar el número total de efectivos se evidencia una mínima cantidad de casos de personal con pérdida auditiva, que inclusive, puede ser corregida con ayuda de otoamplífonos o dispositivos especiales.

Debido a que los casos aislados de mayor pérdida auditiva se presentan en aerotécnicos a cargo de actividades de mantenimiento de aeronaves de ala rotatoria y supersónica, es necesario profundizar en estudios audiométricos más frecuentes y detallados en el personal desplegado en las instalaciones destinadas para este tipo de aviones.

Declaración de conflicto de interés: El autor no manifiesta conflictos de interés institucionales ni personales.

\section{Referencias}

Álvarez-Fernández, C., Romero-Saldaña, M., \& Prieto-Ballesteros, A. (2016). Evaluación epidemiológica de la exposición a ruido en trabajadores de policía local y bomberos. Revista de la Asociación Española de Especialistas en Medicina del Trabajo, 25(2), 86-95. https://scielo.isciii.es/scielo. php?script=sci_arttext\&pid=S1132-62552016000200004

Caputo, L., \& Correa, M. A. (2018). Manejo del ruido en las tripulaciones de la Fuerza Aérea Colombiana en las últimas dos décadas. Ciencia y Poder Aéreo, 13(1), 46-53. https:// doi.org/10.18667/cienciaypoderaereo.585

Carpio-Ayora, M. X., \& Álvarez-Pesantez, K. del R. (2017). Estudio transversal: hipoacusia laboral inducida por ruido en personal de aeronáutica del ejército ecuatoriano y factores asociados. Quito, Ecuador, 2014-2016. Revista Médica Hospital del José Carrasco Arteaga, 9(2), 116-122. https://doi.org/10.14410/2017.9.2.ao.19
Coello, F., Males, D., Tello, D., \& López-Pulles, R. (2017). ¿Son los tapones auditivos eficaces para prevenir los efectos de la contaminación auditiva mediante la atenuación sonora? Revista de la Facultad de Ciencias Médicas (Quito), 42(1), 108-113. https://doi.org/10.29166/ciencias_ medicas.v42i1.1541

Corbalán, E., Trujillo, C., Sznajderman, L., \& Di Bernardi, C. A. (2019). Interacción del aeropuerto de la plata con su entorno a través de mapas estratégicos de ruido y mapas de dispersión gaseosa [Ponencia]. V Jornadas de Investigación, Transferencia y Extensión de la Facultad de Ingeniería, La Plata, Argentina. http://sedici.unlp.edu.ar/ handle/10915/74993

Gordon, J. S., Griest, S. E., Thielman, E. J., Carlson, K. F., Helt, W. J., Lewis, M. S., Blankenship, C., Austin, D., Theodoroff, S. M., \& Henry, J. A. (2017). Audiologic characteristics in a sample of recently-separated military veterans: The Noise Outcomes in Servicemembers Epidemiology Study (NOISE Study). Hearing Research, 349, 21-30. https://doi.org/10.1016/j.heares.2016.11.014

Gwenolé NEXER. (2013). Dans quelle situation l'utilisation d'une double protection auditive devient-elle nécessaire?. Hearing Protech. https://www.hearingprotech.com/fr/publications/doble-protection-auditive.html

Hecht, Q. A., Hammill, T. L., Calamia, P. T., Smalt, C. J., \& Brungart, D. S. (2019). Characterization of acute hearing changes in United States military populations. The Journal of the Acoustical Society of America, 146(5), 3.8393.848. https://doi.org/10.1121/1.5132710

Hernández-Sánchez, H. (2013). Medio militar y trastornos auditivos inducidos por ruido. Revista Cubana de Medicina Militar, 42(3), 396-402. http://scielo.sld.cu/scielo. php?script=sci_arttext\&pid=S0138-65572013000300006

Héroux, M. E., Babisch, W., Belojevic, G., Brink, M., Janssen, S., Lercher, P., Paviotti, M., Pershagen, G., Waye, K. P., Preis, A., Stansfeld, S., Van den Berg, M., \& Verbeek, J. (2018). WHo Environmental Noise Guidelines for the European Region: A Systematic Review on Environmental Noise and Cardiovascular and Metabolic Effects: A Summary. International Journal of Environmental Research and Public Health, 15(2), 379. https://doi.org/10.3390/ ijerph15020379

Heupa, A. B., Gonçalves, C. G. de O., \& Coifman, H. (2011). Effects of impact noise on the hearing of military personnel. Brazilian Journal of Otorhinolaryngology, 77(6), 747753. https://doi.org/10.1590/s1808-86942011000600011 
Irgens-Hansen, K., Sunde, E., Bråtveit, M., Baste, V., Oftedal, G., Koefoed, V., Lind, O., \& Moen, B. E. (2015). Hearing loss in the royal Norwegian navy: a cross-sectional study. International Archives of Occupational and Environmental Health, 88(5), 641-649. https://doi.org/10.1007/ s00420-014-0988-8

Kerr, M. J., Neitzel, R. L., Hong, O. S., \& Sataloff, R. T. (2017). Historical review of efforts to reduce noise-induced hearing loss in the United States. American Journal of Industrial Medicine, 60(6), 569-577. https://doi.org/10.1002/ ajim. 22627

Ministerio de Trabajo y Asuntos Sociales. (2000, 26 de enero). [Real Decreto 1971/1999]. De procedimiento para el reconocimiento, declaración y calificación del grado de minusvalía. BOE: número 22. https://www.boe.es/eli/es/ rd/1999/12/23/1971/con

Olarieta, J., García-Alcántara, F., Pérez, N., \& Rivera, T. (2015). Hipoacusia. Mediciene, 11(91), 5445-5454. https://doi. org/10.1007/978-88-470-1649-1_43

Rodríguez, O. A. (2003). Determinación de aptitud de protectores auditivos. http://www.siafa.com.ar/notisiafa/353/ auditivos.pdf
Stolk, C., Staetsky, L., Hassan, E., \& Kim, C. W. (2012). Management of occupational safety and health: An Analysis of the findings of the European Survey of Enterprises on New and Emerging Risks (ESENER). European Agency for Safety and Health at Work (Vol. 3). https://osha. europa.eu/en/tools-and-publications/publications/ reports/management-of-occupational-safety-and-healthanalysis-of-data-from-the-esener/view

Tepe, V., Smalt, C., Nelson, J., Quatieri, T., \& Pitts, K. (2017). Hidden hearing injury: The emerging science and military relevance of cochlear synaptopathy. Military Medicine, 182(9), e1785-e1795. https://doi.org/10.7205/ MILMED-D-17-00025

Wells, T. S., Seelig, A. D., Ryan, M. A., Jones, J. M., Hooper, T. I., Jacobson, I. G., \& Boyko, E. J. (2015). Hearing loss associated with us military combat deployment. Noise and Health, 17(74), 34-42. https://doi.org/10.4103/1463-17 41.149574

Yankaskas, K., Hammill, T., Packer, M., \& Zuo, J. (2017). Editorial: Auditory injury. A military perspective. Hearing Research, 349, 1-3. https://doi.org/10.1016/j.heares.2017. 04.010 